\title{
Randić Color Energy of a Graph
}

\author{
P. Rajendra \\ Department of Mathematics, Bharathi College, PG and Research Centre \\ Bharathinagara, Mandya - 571422 \\ India
}

\section{ABSTRACT}

Let $G=(V, E)$ be a colored graph with vertex set $V(G)$ and edge set $E(G)$ with chromatic number $\chi(G)$ and $d_{i}$ is the degree of a vertex $v_{i}$. The Randić matrix $R(G)=\left(r_{i j}\right)$ of a graph $G$, is defined by $r_{i j}=\frac{1}{\sqrt{d_{i} d_{j}}}$, if the vertices $v_{i}$ and $v_{j}$ are adjacent and $r_{i j}=0$, otherwise. The Randic energy [5] $R E(G)$ is the sum of absolute values of the eigenvalues of $R(G)$. The concept of Randić color energy $E_{R C}(G)$ of a colored graph $G$ is defined and obtained the Randic color energy $E_{R C}(G)$ of some graphs with minimum number of colors.

\section{Keywords}

Colored graph, Randić matrix, Randić color energy

\section{INTRODUCTION}

Let $G=(V, E)$ be a graph on vertex set $V(G)=\left\{v_{1}, v_{2}, \ldots, v_{n}\right\}$ and edge set $E(G)$. Let $d_{i}$ be the degree of a vertex $v_{i}$ for $i=1,2, \ldots, n$. The adjacency matrix $A(G)=\left(a_{i j}\right)$ of a graph $G$ is a square matrix of order $n$, where

$$
\left(a_{i j}\right)= \begin{cases}1, & \text { if the vertices } v_{i} \text { and } v_{j} \text { are adjacent; } \\ 0, & \text { otherwise. }\end{cases}
$$

Since $A(G)$ is symmetric, its eigenvalues $\lambda_{1}, \lambda_{2}, \ldots, \lambda_{n}$ are all real numbers, whose sum is equal to zero. The energy $E(G)[14]$ of a graph $G$ is

$$
E(G)=\sum_{i=1}^{n}\left|\lambda_{i}\right|
$$

In 1975 by Milan Randić [10], is defined Randić index as $R=R(G)=\sum_{i \sim j} \frac{1}{d_{i} d_{j}}$, where $\sum_{i \sim j}$ indicates summation over all pairs of adjacent vertices $v_{i}$ and $v_{j}$. Randić matrix [5] $R(G)=\left(r_{i j}\right)$ of $G$ is a $n \times n$ symmetric matrix defined by

$$
r_{i j}= \begin{cases}0, & i=j ; \\ \frac{1}{\sqrt{d_{i} d_{j}}}, & v_{i} \text { and } v_{j} \text { are adjacent; } \\ 0, & v_{i} \text { and } v_{j} \text { are not adjacent. }\end{cases}
$$

Let $\rho_{1}, \rho_{2}, \ldots, \rho_{n}$ be the eigenvalues of the Randić matrix $R(G)$, these eigenvalues are real numbers, and that their sum is zero, the Randic energy [5] of a graph $G$ is defined as

$$
R E=R E(G)=\sum_{i=1}^{n}\left|\rho_{i}\right| .
$$

In the last few years, research publications on Randić spectrum, Randić indices and Randić energy can be found in literature [3], [4], [5], [7], [8].

A coloring of graph [1] $G$ is a coloring of its vertices such that no two adjacent vertices receive the same color. The minimum number of colors needed for coloring of a graph $G$ is called chromatic number and denoted by $\chi(G)$. The color adjacency matrix [1] $A_{C}(G)$ are as follows: If $c\left(v_{i}\right)$ is the color of $v_{i}$, then

$$
a_{i j}= \begin{cases}1, & \text { if } v_{i} \text { and } v_{j} \text { are adjacent with } c\left(v_{i}\right) \neq c\left(v_{j}\right) \\ -1, & \text { if } v_{i} \text { and } v_{j} \text { are non-adjacent with } c\left(v_{i}\right)=c\left(v_{j}\right) \\ 0, & \text { otherwise. }\end{cases}
$$

If $\lambda_{1}, \lambda_{2}, \ldots, \lambda_{n}$ are eigenvalues of $A_{c}(G)$ are called color eigenvalues. Color energy [1] of a graph is

$$
E_{c}(G)=\sum_{i=1}^{n}\left|\lambda_{i}\right|
$$

For more literature on color energy of a graph, we can see [1], [2], [11], [12], [13].

\subsection{Randic color matrix and Randić color energy}

Motivated by Color Energy of a Graph [1] and Randić Matrix and Randić Energy [5], have obtained a new matrix called Randić color matrix.

Let $G$ be a simple colored graph with $n$ vertices. The Randić color matrix $A_{R C}(G)=\left(r_{i j}\right)$ is a square $n \times n$ matrix defined by

$r_{i j}= \begin{cases}\frac{1}{\sqrt{d_{i} d_{j}}}, & \text { if } v_{i} \text { and } v_{j} \text { are adjacent with } c\left(v_{i}\right) \neq c\left(v_{j}\right) ; \\ \frac{-1}{\sqrt{d_{i} d_{j}}}, & \text { if } v_{i} \text { and } v_{j} \text { are non-adjacent with } c\left(v_{i}\right)=c\left(v_{j}\right) ; \\ 0, & \text { otherwise. }\end{cases}$

The characteristic polynomial of $A_{R C}(G)$ is $\left|\rho I-A_{R C}(G)\right|$. Let $\rho_{1}, \rho_{2}, \ldots, \rho_{n}$ be eigenvalues of Randić color matrix $A_{R C}(G)$. Since $A_{R C}(G)$ is real and symmetric matrix, so its eigenvalues are real numbers and that their sum is zero. If the eigenvalues of $A_{R C}(G)$ are $\rho_{1}, \rho_{2}, \ldots, \rho_{n}$ with their multiplicities 
are $m_{1}, m_{2}, \ldots, m_{r}$ then spectrum of $A_{R C}(G)$ is denoted by

$$
\operatorname{Spec}_{R C}(G)=\left(\begin{array}{ccccc}
\rho_{1} & \rho_{2} & \ldots & \rho_{n-1} & \rho_{n} \\
m_{1} & m_{2} & \ldots & m_{r-1} & m_{r}
\end{array}\right) .
$$

The Randić color energy $E_{R C}(G)$ of a colored graph $G$ is defined as

$$
E_{R C}(G)=\sum_{i=1}^{n}\left|\rho_{i}\right|
$$

\section{RANDI $C ́$ COLOR ENERGY OF SOME GRAPHS}

THEOREM 1. If $K_{n}$ is complete graph with $n$ vertices and $\chi\left(K_{n}\right)=n$, then Randic color energy of $K_{n}$ is $E_{R C}\left(K_{n}\right)=2$.

Proof. Let $K_{n}$ be the complete graph with $n$ vertices. Since $\chi\left(K_{n}\right)=n$, we have Randić color matrix

$$
\left|\rho I-A_{R C}\left(K_{n}\right)\right|=\left|\begin{array}{ccccccc}
\rho & \frac{-1}{n-1} & \frac{-1}{n-1} & \ldots & \frac{-1}{n-1} & \frac{-1}{n-1} & \frac{-1}{n-1} \\
\frac{-1}{n-1} & \rho & \frac{-1}{n-1} & \ldots & \frac{-1}{n-1} & \frac{-1}{n-1} & \frac{-1}{n-1} \\
\frac{-1}{n-1} & \frac{-1}{n-1} & \rho & \ldots & \frac{-1}{n-1} & \frac{-1}{n-1} & \frac{-1}{n-1} \\
\vdots & \vdots & \vdots & \ddots & \vdots & \vdots & \vdots \\
\frac{-1}{n-1} & \frac{-1}{n-1} & \frac{-1}{n-1} & \cdots & \rho & \frac{-1}{n-1} & \frac{-1}{n-1} \\
\frac{-1}{n-1} & \frac{-1}{n-1} & \frac{-1}{n-1} & \ldots & \frac{-1}{n-1} & \rho & \frac{-1}{n-1} \\
\frac{-1}{n-1} & \frac{-1}{n-1} & \frac{-1}{n-1} & \ldots & \frac{-1}{n-1} & \frac{-1}{n-1} & \rho
\end{array}\right|_{n \times n}
$$

$R_{1}^{\prime}=R_{1}+R_{2}+\cdots+R_{n-1}+R_{n}$, then we get $(\rho-1)$ common in first row

$\left|\rho I-A_{R C}\left(K_{n}\right)\right|=(\rho-1)\left|\begin{array}{ccccccc}1 & 1 & 1 & \ldots & 1 & 1 & 1 \\ \frac{-1}{n-1} & \rho & \frac{-1}{n-1} & \ldots & \frac{-1}{n-1} & \frac{-1}{n-1} & \frac{-1}{n-1} \\ \frac{-1}{n-1} & \frac{-1}{n-1} & \rho & \ldots & \frac{-1}{n-1} & \frac{-1}{n-1} & \frac{-1}{n-1} \\ \vdots & \vdots & \vdots & \ddots & \vdots & \vdots & \vdots \\ \frac{-1}{n-1} & \frac{-1}{n-1} & \frac{-1}{n-1} & \cdots & \rho & \frac{-1}{n-1} & \frac{-1}{n-1} \\ \frac{-1}{n-1} & \frac{-1}{n-1} & \frac{-1}{n-1} & \cdots & \frac{-1}{n-1} & \rho & \frac{-1}{n-1} \\ \frac{-1}{n-1} & \frac{-1}{n-1} & \frac{-1}{n-1} & \cdots & \frac{-1}{n-1} & \frac{-1}{n-1} & \rho\end{array}\right|_{n \times n}$

$R_{k}^{\prime}=R_{k}+\frac{R_{1}}{n-1}, k=2,3, \ldots, n$, we get, Characteristic polynomial $\left|\rho I-A_{R C}\left(K_{n}\right)\right|=(\rho-1)\left(\rho+\frac{1}{n-1}\right)^{n-1}$,

$\operatorname{Spec}_{R C}\left(K_{n}\right)=\left(\begin{array}{cc}\frac{-1}{n-1} & 1 \\ n-1 & 1\end{array}\right)$, Randić Color Energy of $K_{n}$ is $E_{R C}\left(K_{n}\right)=2$.

THEOREM 2. If $S_{n}$ is star graph with $n$ vertices and $\chi\left(S_{n}\right)=2$, then Randić color energy of $S_{n}$ is $E_{R C}\left(S_{n}\right)=\sqrt{n^{2}-4 n+8}+(n-2)$.
Proof. Let $S_{n}$ be the star graph with $n$ vertices. Since $\chi\left(S_{n}\right)=2$, we have Randić color matrix

$\left|\rho I-A_{R C}\left(S_{n}\right)\right|=\left|\begin{array}{ccccccc}\rho & \frac{-1}{\sqrt{n-1}} & \frac{-1}{\sqrt{n-1}} & \ldots & \frac{-1}{\sqrt{n-1}} & \frac{-1}{\sqrt{n-1}} & \frac{-1}{\sqrt{n-1}} \\ \frac{-1}{\sqrt{n-1}} & \rho & 1 & \ldots & 1 & 1 & 1 \\ \frac{-1}{\sqrt{n-1}} & 1 & \rho & \ldots & 1 & 1 & 1 \\ \vdots & \vdots & \vdots & \ddots & \vdots & \vdots & \vdots \\ \frac{-1}{\sqrt{n-1}} & 1 & 1 & \ldots & \rho & 1 & 1 \\ \frac{-1}{\sqrt{n-1}} & 1 & 1 & \ldots & 1 & \rho & 1 \\ \frac{-1}{\sqrt{n-1}} & 1 & 1 & \ldots & 1 & 1 & \rho\end{array}\right|_{n \times n}$

$R_{k}^{\prime}=R_{k}-R_{k-1}, k=3,4,5, \ldots,(n-1), n$, then taking $(\rho-1)$ common from $R_{3}$ to $R_{n}$, we get

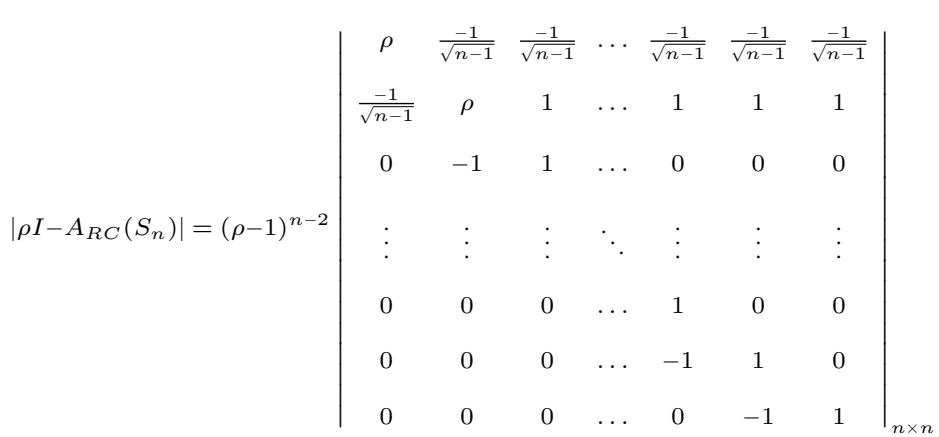

$C_{k}^{\prime}=C_{k}+C_{k+1}+\cdots+C_{n}, k=2,3, \ldots,(n-1), n$, then

$$
\left|\rho I-A_{R C}\left(S_{n}\right)\right|=(\rho-1)^{n-2}\left|\begin{array}{cc}
\rho & \frac{-(n-1)}{\sqrt{n-1}} \\
\frac{-1}{\sqrt{n-1}} & \rho+(n-2)
\end{array}\right|,
$$

Characteristic polynomial

$$
\begin{gathered}
\left|\rho I-A_{R C}\left(S_{n}\right)\right|=(\rho-1)^{n-2}\left[\rho^{2}+(n-2) \rho-1\right], \\
\operatorname{Spec}_{R C}\left(S_{n}\right)=\left(\begin{array}{ccc}
\frac{-(n-2)-\sqrt{n^{2}-4 n+8}}{2} & \frac{-(n-2)+\sqrt{n^{2}-4 n+8}}{2} & 1 \\
1 & 1 & n-2
\end{array}\right),
\end{gathered}
$$

Randić Color Energy of $S_{n}$ is

$$
E_{R C}\left(S_{n}\right)=\sqrt{n^{2}-4 n+8}+(n-2) .
$$

THEOREM 3. If $K_{n, n}$ is complete bipartite graph with $2 n$ vertices and $\chi\left(K_{n, n}\right)=2$, then Randic color energy of $K_{n, n}$ is $E_{R C}\left(K_{n, n}\right)=\frac{4 n-2}{n}$.

Proof. Let $K_{n, n}$ be the complete bipartite graph with $2 n$ vertices. Since $\chi\left(K_{n, n}\right)=2$, we have Randić color matrix 
$\left|\rho I-A_{R C}\left(K_{n, n}\right)\right|=\left|\begin{array}{cccccccccc}\rho & \frac{1}{n} & \ldots & \frac{1}{n} & \frac{1}{n} & \frac{-1}{n} & \frac{-1}{n} & \ldots & \frac{-1}{n} & \frac{-1}{n} \\ \frac{1}{n} & \rho & \ldots & \frac{1}{n} & \frac{1}{n} & \frac{-1}{n} & \frac{-1}{n} & \ldots & \frac{-1}{n} & \frac{-1}{n} \\ \vdots & \vdots & \ddots & \vdots & \vdots & \vdots & \vdots & \ddots & \vdots & \vdots \\ \frac{1}{n} & \frac{1}{n} & \ldots & \rho & \frac{1}{n} & \frac{-1}{n} & \frac{-1}{n} & \ldots & \frac{-1}{n} & \frac{-1}{n} \\ \frac{1}{n} & \frac{1}{n} & \ldots & \frac{1}{n} & \rho & \frac{-1}{n} & \frac{-1}{n} & \ldots & \frac{-1}{n} & \frac{-1}{n} \\ \frac{-1}{n} & \frac{-1}{n} & \ldots & \frac{-1}{n} & \frac{-1}{n} & \rho & \frac{1}{n} & \ldots & \frac{1}{n} & \frac{1}{n} \\ \frac{-1}{n} & \frac{-1}{n} & \ldots & \frac{-1}{n} & \frac{-1}{n} & \frac{1}{n} & \rho & \ldots & \frac{1}{n} & \frac{1}{n} \\ \vdots & \vdots & \ddots & \vdots & \vdots & \vdots & \vdots & \ddots & \vdots & \vdots \\ \frac{-1}{n} & \frac{-1}{n} & \ldots & \frac{-1}{n} & \frac{-1}{n} & \frac{1}{n} & \frac{1}{n} & \ldots & \rho & \frac{1}{n} \\ \frac{-1}{n} & \frac{-1}{n} & \ldots & \frac{-1}{n} & \frac{-1}{n} & \frac{1}{n} & \frac{1}{n} & \ldots & \frac{1}{n} & \rho\end{array}\right|_{2 n \times 2 n}$

$R_{1}^{\prime}=\left(R_{1}+R_{2}+\cdots+R_{n}\right)-\left(R_{n+1}+R_{n+2}+\cdots+R_{2 n}\right)$, $R_{k}^{\prime}=R_{k}+R_{2 n}, k=2,3, \ldots, n$ and $R_{k}^{\prime}=R_{k}-R_{2 n}$, $k=n+1, n+2, \ldots, 2 n-1$, then we get $\left(\rho+\frac{2 n-1}{n}\right)$ common in first row and $\left(\rho-\frac{1}{n}\right)$ common from $R_{2}$ to $R_{2 n-1}$

$=\left(\rho+\frac{2 n-1}{n}\right)\left(\rho-\frac{1}{n}\right)^{2 n-2}\left|\begin{array}{cccccccccc}1 & 1 & \ldots & 1 & 1 & -1 & -1 & \ldots & -1 & -1 \\ 0 & 1 & \ldots & 0 & 0 & 0 & 0 & \ldots & 0 & 1 \\ \vdots & \vdots & \ddots & \vdots & \vdots & \vdots & \vdots & \ddots & \vdots & \vdots \\ 0 & 0 & \ldots & 1 & 0 & 0 & 0 & \ldots & 0 & \vdots \\ 0 & 0 & \ldots & 0 & 1 & 0 & 0 & \ldots & 0 & 1 \\ 0 & 0 & \ldots & 0 & 0 & 1 & 0 & \ldots & 0 & -1 \\ 0 & 0 & \ldots & 0 & 0 & 0 & 1 & \ldots & 0 & -1 \\ \vdots & \vdots & \ddots & \vdots & \vdots & \vdots & \vdots & \ddots & \vdots & \vdots \\ 0 & 0 & \ldots & 0 & 0 & 0 & 0 & \ldots & \vdots & -1 \\ \frac{-1}{n} & \frac{-1}{n} & \ldots & \frac{-1}{n} & \frac{-1}{n} & \frac{1}{n} & \frac{1}{n} & \ldots & \frac{1}{n} & \rho\end{array}\right|_{2 n \times 2 n}$

$R_{2 n}^{\prime}=R_{2 n}+\frac{R_{1}}{n}$, we get Characteristic polynomial

$$
\begin{aligned}
\left|\rho I-A_{R C}\left(K_{n, n}\right)\right| & =\left(\rho+\frac{2 n-1}{n}\right)\left(\rho-\frac{1}{n}\right)^{2 n-1}, \\
\operatorname{Spec}_{R C}\left(K_{n, n}\right) & =\left(\begin{array}{cc}
-\left(\frac{2 n-1}{n}\right) & \frac{1}{n} \\
1 & 2 n-1
\end{array}\right) .
\end{aligned}
$$

Randić Color Energy of $K_{n, n}$ is $E_{R C}\left(K_{n, n}\right)=\frac{4 n-2}{n}$.

THEOREM 4. If $K_{n, n+1}$ is complete bipartite graph with $2 n+1$ vertices and $\chi\left(K_{n, n+1}\right)=2$, then Randić color energy of $K_{n, n+1}$ is

$$
E_{R C}\left(K_{n, n+1}\right)=\frac{2\left[\sqrt{n^{2}+2 n+2}+n\right]}{n+1} .
$$

PROOF. Let $K_{n, n+1}$ be the complete bipartite graph with $2 n+1$ vertices. Since $\chi\left(K_{n, n+1}\right)=2$, we have Randić color matrix $A_{R C}\left(K_{n, n+1}\right)$ is a square matrix of order $2 n+1$.

$$
\left|\rho I-A_{R C}\left(K_{n, n+1}\right)\right|=
$$

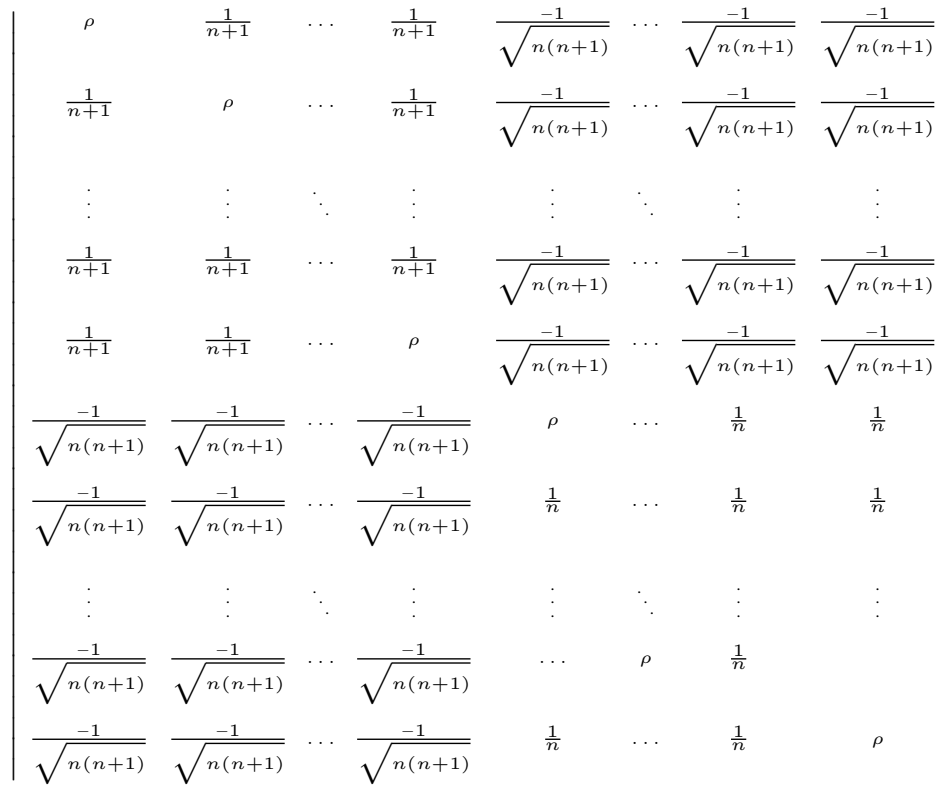

$R_{k}^{\prime}=R_{k}-R_{n}, k=1,2,3, \ldots, n-1, R_{m}^{\prime}=R_{m}-R_{n+1}$, $m=n+2, n+3, \ldots, 2 n+1$ then taking $\left(\rho-\frac{1}{n+1}\right)$ common from $R_{1}$ to $R_{n-1}$ and $\left(\rho-\frac{1}{n}\right)$ from $R_{n+2}$ to $R_{2 n+1}$

$$
\left(\rho-\frac{1}{n+1}\right)^{n-1}\left(\rho-\frac{1}{n}\right)^{n}
$$

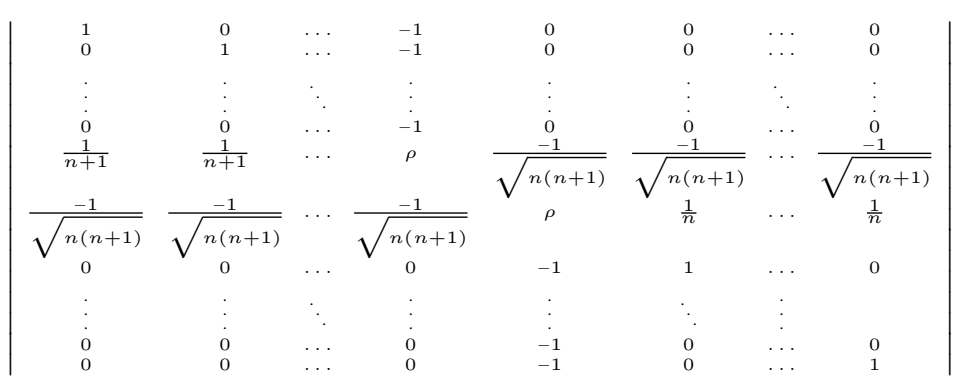
$C_{n+1}^{\prime}=C_{n+1}+C_{n+2}+\cdots+C_{2 n+1}$

$$
=\left(\rho-\frac{1}{n+1}\right)^{n-1}\left(\rho-\frac{1}{n}\right)^{n}
$$

$\left|\begin{array}{cccccc}1 & 0 & \cdots & 0 & -1 & 0 \\ 0 & 1 & \cdots & 0 & -1 & 0 \\ \vdots & \vdots & \ddots & \vdots & \vdots & \vdots \\ 0 & 0 & \cdots & 1 & -1 & 0 \\ \frac{1}{n+1} & \frac{1}{n+1} & \cdots & \frac{1}{n+1} & \rho & \frac{-(n+1)}{\sqrt{n(n+1)}} \\ \frac{-1}{\sqrt{n(n+1)}} & \frac{-1}{\sqrt{n(n+1)}} & \cdots & \frac{-1}{\sqrt{n(n+1)}} \frac{-1}{\sqrt{n(n+1)}} & \rho+1\end{array}\right|_{(n+1) \times(n+1)}$


$C_{n}^{\prime}=C_{1}+C_{2}+\cdots+C_{n-1}+C_{n}$, then

$\left|\rho I-A_{R C}\left(K_{n, n+1}\right)\right|=\left(\rho-\frac{1}{n+1}\right)^{n-1}\left(\rho-\frac{1}{n}\right)^{n} \mid \begin{array}{cc}\rho+\frac{n-1}{n+1} & \frac{-(n+1)}{\sqrt{n(n+1)}} \\ \frac{-n}{\sqrt{n(n+1)}} & \rho+1\end{array}$

Characteristic polynomial

$\left|\rho I-A_{R C}\left(K_{n, n+1}\right)\right|=\left(\rho-\frac{1}{n+1}\right)^{n-1}\left(\rho-\frac{1}{n}\right)^{n}\left[(n+1) \rho^{2}+2 n \rho-2\right]$,

$\operatorname{Spec}_{R C}\left(K_{n, n+1}\right)=\left(\begin{array}{cccc}\frac{-n-\sqrt{n^{2}+2 n+2}}{n+1} & \frac{-n+\sqrt{n^{2}+2 n+2}}{n+1} & \frac{1}{n+1} & \frac{1}{n} \\ 1 & 1 & n-1 & n\end{array}\right)$,

Randić Color Energy of $K_{n, n+1}$ is

$$
E_{R C}\left(K_{n, n+1}\right)=\frac{2\left[\sqrt{n^{2}+2 n+2}+n\right]}{n+1} .
$$

THEOREM 5. If $K_{m, n}$ is complete bipartite graph with $m+n$ vertices and $\chi\left(K_{m, n}\right)=2$, then Randic color energy of $K_{m, n}$ is

$E_{R C}\left(K_{m, n}\right)=\frac{\sqrt{\left(m^{2}+n^{2}-m-n\right)^{2}+4 m n(m+n-1)}+\left(m^{2}+n^{2}-m-n\right)}{m n}$,

where $m<n$.

PROOF. Let $K_{m, n}$ be the complete bipartite graph with $m+n$ vertices. Since $\chi\left(K_{m, n}\right)=2$, we have Randić color matrix

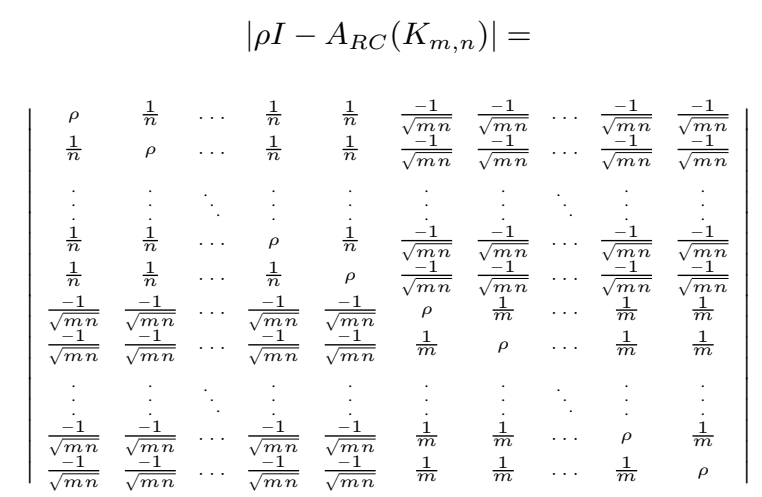

$R_{k}^{\prime}=R_{k}-R_{m}, k=1,2,3, \ldots, m-1, R_{d}^{\prime}=R_{d}-R_{m+1}$, $d=m+2, m+3, \ldots, m+n$, then taking $\left(\rho-\frac{1}{n}\right)$ common from $R_{1}$ to $R_{m-1}$ and taking $\left(\rho-\frac{1}{m}\right)$ common from $R_{m+2}$ to $R_{m+n}$, we get

$$
\left(\rho-\frac{1}{n}\right)^{m-1}\left(\rho-\frac{1}{m}\right)^{n-1}
$$

$$
\left|\begin{array}{cccccccccc}
1 & 0 & \cdots & 0 & -1 & 0 & 0 & \cdots & 0 & 0 \\
0 & 1 & \cdots & 0 & -1 & 0 & 0 & \cdots & 0 & 0 \\
\vdots & \vdots & \ddots & \vdots & \vdots & \vdots & \vdots & \ddots & \vdots & \vdots \\
0 & 0 & \cdots & 1 & -1 & 0 & 0 & \cdots & 0 & 0 \\
\frac{1}{n} & \frac{1}{n} & \cdots & \frac{1}{n} & \rho & \frac{-1}{\sqrt{m n}} & \frac{-1}{\sqrt{m n}} & \cdots & \frac{-1}{\sqrt{m n}} & \frac{-1}{\sqrt{m n}} \\
\frac{-1}{\sqrt{m n}} & \frac{-1}{\sqrt{m n}} & \cdots & \frac{-1}{\sqrt{m n}} & \frac{-1}{\sqrt{m n}} & \rho & \frac{1}{m} & \cdots & \frac{1}{m} & \frac{1}{m} \\
0 & 0 & \cdots & 0 & 0 & -1 & 1 & \cdots & 0 & 0 \\
\vdots & \vdots & \ddots & \vdots & \vdots & \vdots & \vdots & \ddots & \vdots & \vdots \\
0 & 0 & \cdots & 0 & 0 & -1 & 0 & \cdots & 1 & 0 \\
0 & 0 & \cdots & 0 & 0 & -1 & 0 & \cdots & 0 & 1
\end{array}\right|
$$

Characteristic polynomial

$\left|\rho I-A_{R C}\left(K_{m, n}\right)\right|=\left(\rho-\frac{1}{n}\right)^{m-1}\left(\rho-\frac{1}{m}\right)^{n-1}\left[m n \rho^{2}+\left(n^{2}+m^{2}-n-m\right) \rho-(m+n-1)\right]$

$\operatorname{Spec}_{R C}(K m, n)=\left[\begin{array}{ccc}\frac{-\left(m^{2}+n^{2}-m-n\right) \pm \sqrt{\left(m^{2}+n^{2}-m-n\right)^{2}+4 m n(m+n-1)}}{2 m n} & \frac{1}{n} & \frac{1}{m} \\ 1 & m-1 & n-1\end{array}\right]$

Randić Color Energy of $K_{m, n}$ is

$E_{R C}\left(K_{m, n}\right)=\frac{\sqrt{\left(m^{2}+n^{2}-m-n\right)^{2}+4 m n(m+n-1)}+\left(m^{2}+n^{2}-m-n\right)}{m n}$.

THEOREM 6. If $S_{n}^{0}$ is crown graph with $2 n$ vertices and $\chi\left(S_{n}^{0}\right)=2$, then Randic color energy of $S_{n}^{0}$ is $E_{R C}\left(S_{n}^{0}\right)=\frac{4 n-4}{n}$.

Proof. Let $S_{n}^{0}$ be the crown graph with $2 n$ vertices. Since $\chi\left(S_{n}^{0}\right)=2$, we have Randić color matrix

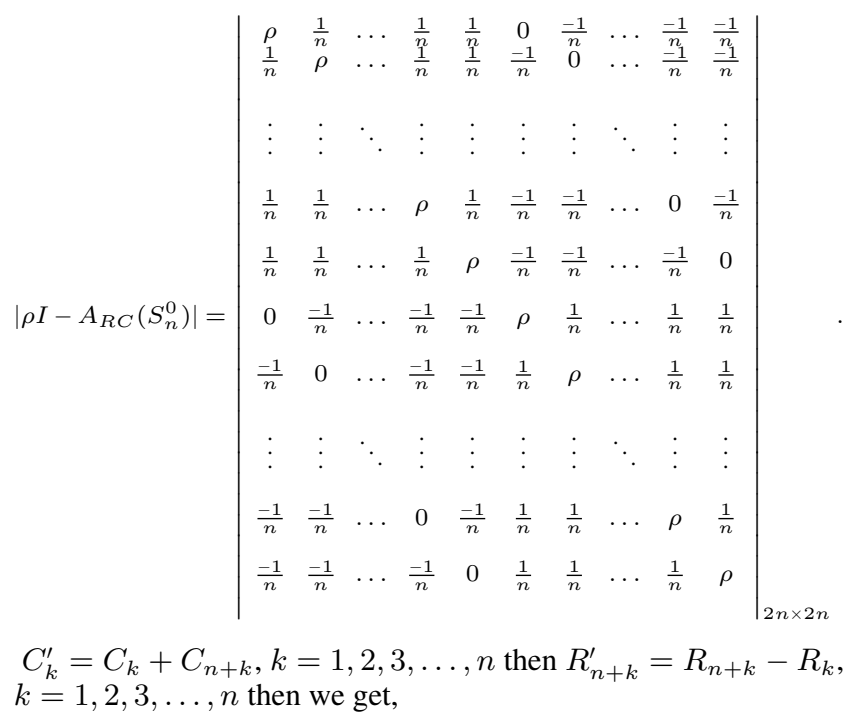

$$
\left|\rho I-A_{R C}\left(S_{n}^{0}\right)\right|=\rho^{n}\left|\begin{array}{ccccc}
\rho & \frac{2}{n} & \ldots & \frac{2}{n} & \frac{2}{n} \\
\frac{2}{n} & \rho & \ldots & \frac{2}{n} & \frac{2}{n} \\
\vdots & \vdots & \ddots & \vdots & \vdots \\
\frac{2}{n} & \frac{2}{n} & \ldots & \rho & \frac{2}{n} \\
\frac{2}{n} & \frac{2}{n} & \ldots & \frac{2}{n} & \rho
\end{array}\right|_{n \times n}
$$

Characteristic polynomial

$$
\begin{gathered}
\left|\rho I-A_{R C}\left(S_{n}^{0}\right)\right|=\rho^{n}\left(\rho+\frac{2(n-1)}{n}\right)\left(\rho-\frac{2}{n}\right)^{n-1}, \\
\operatorname{Spec}_{R C}\left(S_{n}^{0}\right)=\left(\begin{array}{ccc}
-\left(\frac{2(n-1)}{n}\right) & 0 & \frac{2}{n} \\
1 & n & n-1
\end{array}\right) .
\end{gathered}
$$

Randić Color Energy of $S_{n}^{0}$ is $E_{R C}\left(S_{n}^{0}\right)=\frac{4(n-1)}{n}$. 


\section{ACKNOWLEDGEMENT:}

The author would like to thank the Prof. R. Rangarajan, DOS in Mathematics, University of Mysore, Mysuru, for many useful suggestions.

\section{REFERENCES}

[1] C. Adiga, E. Sampathkumar and M. A. Sriraj, Color Energy of a Graph, Proc. Jangjeon Math. Soc., 16 (3), (2013), 335-351.

[2] C. Adiga, E. Sampathkumar and M. A. Sriraj, Color Energy of a Unitary Cayley Graph, Discussiones Mathematicae Graph Theory, 34 (2014), 707-721.

[3] Bolian Liu, Yufei Huang and Jingfang Feng, A Note on the Randić Spectral Radius, MATCH Commun. Math. Comput. Chem., 68 (2012), 913-916.

[4] Ş. Burcu Bozkurt, A. Dilek Güngör and Ivan Gutman, Randić Spectral Radius and Randić Energy, MATCH Commun. Math. Comput. Chem., 64 (2010), 321-334.

[5] Ş. Burcu Bozkurt, A. Dilek Güngör, Ivan Gutman and A. Sinan Cevik, Randić Matrix and Randić Energy, MATCH Commun. Math. Comput. Chem., 64 (2010), 239-250.

[6] D. M. Cvetkovic, M. Doob and H. Sachs, Spectra of Graphs, Theory and Application, Academic Press, New York, USA(1980).

[7] K. C. Das and S. Sorgun: On Randić Energy of Graphs, MATCH Commun. Math. Comput. Chem., 72 (2014), 227238.

[8] A. Dilek Maden, New Bounds on the Incidence Energy, Randić Energy and Randić Estrada Index, MATCH Commun. Math. Comput. Chem., 74, (2015), 367-387.

[9] B. Furtula and I. Gutman, Comparing energy and Randić energy, Macedonian Journal of Chemistry and Chemical Engineering, 32(1), (2013), 117-123.

[10] M. Randić, On Characterization of Molecular Branching, J. Am. Chem. Soc., 97 (1975), 6609-6615.

[11] V. S. Shigehalli and Kenchappa S. Betageri, Color Laplacian Energy of Graphs, Journal of Computer and Mathematical Sciences, Vol.6(9), 2015, 485-494.

[12] M.A.Sriraj, Bounds for the Largest Color Eigenvalue and the Color Energy, International J.Math. Combin., Vol.1, (2017), 127-134.

[13] M. A. Sriraj, Some Studies on Energy of Graphs, Ph. D. Thesis, University of Mysore, Mysore, India, 2014.

[14] Xueliang Li, Yongtang Shi and I. Gutman, Graph Energy, Springer New York, 2012. 